\title{
Trois conditions pour une clinique de l'activité en psychologie du travail : le cas d'une intervention dans une entreprise de logistique automobile
}

Three conditions in clinic of activity in work psychology: an intervention in an automobile logistics company

\section{Edwige Quillerou-Grivot et Yves Clot}

\section{OpenEdition \\ Journals}

Édition électronique

URL : http://journals.openedition.org/activites/842

DOI : 10.4000 /activites.842

ISSN : $1765-2723$

Éditeur

ARPACT - Association Recherches et Pratiques sur les ACTivités

\section{Référence électronique}

Edwige Quillerou-Grivot et Yves Clot, «Trois conditions pour une clinique de l'activité en psychologie du travail : le cas d'une intervention dans une entreprise de logistique automobile », Activités [En ligne], 10-2 | Octobre 2013, mis en ligne le 15 octobre 2013, consulté le 10 décembre 2020. URL : http:// journals.openedition.org/activites/842 ; DOI : https://doi.org/10.4000/activites.842

\section{(c) $(7)$}

Activités est mis à disposition selon les termes de la licence Creative Commons Attribution - Pas d'Utilisation Commerciale - Pas de Modification 4.0 International. 


\title{
Trois conditions pour une clinique de l'activité en psychologie du travail : le cas d'une intervention dans une entreprise de logistique automobile
}

\author{
Edwige Quillerou-Grivot
}

Laboratoire Organisation, Changement et Prévention, Homme au Travail, Institut National de Recherche et de Sécurité, 1 rue du Morvan, 54200 Vandoeuvre-les-Nancy

edwige.quillerou@inrs.fr

\section{Yves Clot}

Équipe Psychologie du travail et Clinique de l'activité, Centre de Recherche pour le Travail et le Développement, Conservatoire National des Arts et Métiers, 41 rue Gay Lussac, 75005 Paris

yves.clot@cnam.fr

\begin{abstract}
Three conditions in Activity Clinic in Work Psychology: an Intervention in the Car Industry. This paper is based on an intervention conducted in the Activity Clinic perspective in a subcontractor company in the car industry. The Activity Clinic methodology has been used with bumper assembly operators to support the development of activity and health at work. This paper analyzes the conditions, which allowed the activity clinician's attempts to overcome organizational obstacles and to transform them into new resources for the work development of the operators. We emphasize the necessity for clinical work both with the operators in the form of work co-analysis, and with designers and managers of the firm in the steering committee. It is necessary to maximize opportunities for the development of the activity of the operators and simultaneously, for the transformation of the organizational work, despite the uncertainty of our actions in relation to the complexities of human activity and work organization. On the basis of this analysis, we propose three conditions which seem fundamental to support development of the activity and to spread this analysis throughout the organization. These three conditions are: authenticity of the work dialogue, engagement into controversy, transfers of surprises and affective transfers.. This paper helps helps fuel the debate on the transformation model relating to an Activity Clinic perspective.
\end{abstract}

KEYWORDS

steering committee, health, work co-analysis, intervention, work collective, car industry, development of activity

\section{1.- Introduction}

L'objectif de cet article est à la fois ambitieux et modeste puisqu'il s'agit de poursuivre la réflexion déjà engagée par les chercheurs en clinique de l'activité sur la formalisation de l'intervention et des conditions favorisant le développement de l'activité et du métier (Duboscq, 2009 ; Kostulsk, Clot, \& Litim, 2011 ; Litim, 2012 ; Sannino, 2012 ; Scheller, 2010). Nous souhaitons apporter quelques repères au regard des expériences d'interventions d'une clinique de l'activité en psychologie du travail et spécialement à travers une intervention menée entre 2008 et 2011 chez un logisticien automobile, appartenant à un 
grand groupe industriel. Cette intervention fait suite à une commande émanant du responsable «projets ». Ce dernier souhaitait être mieux informé sur les risques de Troubles Musculo-Squelettiques (TMS) des opérateurs de montage et sur les possibilités d'action des concepteurs sur la prévention de ces risques lors de la mise en place de nouveaux projets.

Dans un premier temps, des éléments concernant la commande, le travail d'opérateurs de montage de pare-chocs et la vie de l'intervention seront présentés. Dans un second temps, le dispositif méthodologique et certains des principaux résultats de cette intervention seront précisés. Enfin, trois conditions cliniques seront identifiées comme favorisant le développement de l'activité des opérateurs, du collectif d'opérateurs et permettant d'agir sur l'organisation du travail. Nous analyserons ainsi cette intervention à travers des éléments précis concernant le dispositif dont se saisit le clinicien de l'activité pour tenter d'agir sur le travail. Nous proposerons une formalisation des enjeux méthodologiques et cliniques au cours de l'intervention.

Bien entendu ces propositions ne représentent pas un mode d'emploi mais plutôt une réflexion sur notre posture clinique dans l'action, dans le quotidien de l'intervention. Il s'agit de comprendre les leviers de notre propre action et d'identifier les questions nouvelles auxquelles nous devons nous confronter dans l'action, puis de reprendre les questions théoriques qu'elles impliquent. Durant l'intervention, les inattendus, les obstacles, les remaniements contribuent à éclairer les situations que nous rencontrons. Ils sont d'ailleurs au cœur de la méthodologie clinique de l'activité en psychologie du travail. Ainsi l'analyse des conditions participant au développement de l'activité, des collectifs et de l'organisation du travail nous permet d'instruire la question des voies possibles du développement d'un métier et de la santé au travail.

\section{2.- L'intervention chez un logisticien automobile}

L'apparition de restrictions médicales et d'arrêts maladies pour des douleurs au niveau des lombaires, des membres supérieurs et parfois même des membres inférieurs est à l'origine d'une demande faite à l'INRS par une entreprise de logistique automobile. Le responsable projets de cette entreprise nous a adressé la question suivante: «à partir de combien de gestes les opérateurs vont-ils avoir des TMS ? ». À ce moment précis de notre intervention, l'entreprise nous faisait part de sa volonté d'intégrer des connaissances sur la prévention des risques professionnels dès la conception de nouveaux projets. À partir d'un premier bilan des conditions de travail, nous leur avions proposé d'engager un travail de co-analyse avec les opérateurs, autour des questions de santé, de gestes professionnels et de l'organisation du travail. L'objectif principal que nous avions établi avec l'entreprise a été de créer un cadre d'analyse du travail favorisant la santé des opérateurs, à partir de questions tant sur la conception que sur la mise en production.

La manière dont on construit le cadre de l'intervention en clinique de l'activité (Clot, 2008a) fait souvent l'objet de recherches du côté de la co-analyse avec les professionnels sur lesquels porte directement la question de métier, de santé, etc. Mais les recherches s'ouvrent du côté des professionnels de l'entreprise qui nous font intervenir, ceux que nous nommons «commanditaires » (ex : membres de direction, concepteurs, CHSCT, etc.). Précisément, lorsqu'on intervient, on crée avec les commanditaires une instance de pilotage, constituée de personnes de l'encadrement, de la direction opérationnelle ou de services fonctionnels. La constitution d'un comité de pilotage permet ainsi de rassembler des membres de l'entreprise qui sont plus ou moins porteuses de la commande et/ou plus ou moins concernées par cette commande. Même si le travail dans l'intervention auprès de ces commanditaires est une préoccupation aussi importante que le travail de co-analyse avec les professionnels, sa formalisation du point de vue de la recherche n'en est qu'à ses débuts. C'est pourquoi, nous tenterons ici d'interroger tant le travail de co-analyse avec les professionnels que celui engagé en comité de pilotage où il est question de faire des choix de transformations des 
situations de travail des opérateurs à plus ou moins long terme.

Cette entreprise de logistique automobile est le dernier maillon de la chaîne de sous-traitance et représente, pour le constructeur automobile situé à quelques kilomètres, un «Magasin Avancé Fournisseur » (MAF) mis à sa disposition par les équipementiers, dans un flux dit «synchrone ». Le travail de montage et d'assemblage de pare-chocs automobiles se réalise, non pas à la chaîne comme chez la plupart des constructeurs automobiles, mais au sein d'un « îlot de production ». Ce principe de production permet une flexibilité à différents niveaux : espace disponible, nombre de machines, nombre d'opérateurs avec exigence de polyvalence à tous les postes, déplacement manuel du produit assuré par les opérateurs, etc. La conception des moyens de travail est répartie entre son client équipementier (qui conçoit les machines, les conteneurs d'arrivée), le constructeur (qui conçoit les conteneurs finaux adaptés à sa propre utilisation) et l'entreprise de logistique (qui adapte la disposition spatiale nécessaire et qui tente d'engager des actions correctives sur les machines, les supports de stocks tampons et les postes de contrôle des pare-chocs).

Les opérateurs réalisent le montage et l'assemblage de composants de pare-chocs automobiles. On observe un cumul d'exigences, liées à des délais rigides (risque d'arrêts de chaîne chez le constructeur avec pénalité financière), à une grande diversité des produits (combinaison d'options, pièces «d'aspect » nécessitant de nombreux critères de qualité et d'esthétisme, etc.), aux impératifs de réactivité, aux aléas et à la flexibilité (polyvalence à tous les postes, apprentissage sur le montage de nouveaux modèles, de nouvelles machines, turn-over important d'intérimaires, etc.). Cette description des multiples exigences du travail ne suffit pas à comprendre le travail et ses enjeux (Hubault \& Bourgeois, 2004). L'intensification est un phénomène complexe (Volkoff, 2008) et ambivalent (Clot, 2005) qui ne peut se résumer aux exigences de la tâche et aux conditions de travail : elle nécessite d'aborder les ressources que les opérateurs développent afin de répondre à ces exigences, tout en préservant leur santé. Cette question des ressources a guidé l'intervention, plus précisément : quelles peuvent être ces ressources ? Comment créer de nouvelles ressources dans cette forme de configuration organisationnelle ? Ces questions font plus généralement l'objet d'interrogations méthodologiques en clinique de l'activité (Scheller, 2010).

Dans ce cadre théorique, les ressources que l'on cherche à développer sont abordées au travers des conflits ou des dilemmes de l'activité de travail, que vit chacun des professionnels. Les conflits de l'activité sont envisagés comme une dynamique propre à l'activité humaine où chacun est confronté à une réalité qui l'oblige constamment à remettre en question ses pré-requis. Ces conflits peuvent être une source de développement pour transformer la situation de travail, ou au contraire devenir un obstacle s'ils ne parviennent pas à être réinvestis dans le travail. Dans l'intervention présentée ici, une co-analyse du travail des opérateurs est proposée afin d'instruire cette question des conflits dans leur activité de travail. C'est particulièrement par la confrontation de leurs gestes professionnels quotidiens à ceux des autres collègues (Clot \& Fernandez, 2005) que nous parvenons à la fois à comprendre les conflits d'activité sous-jacents et à trouver des possibilités de transformation de ces conflits d'activité concernant les situations de travail qui posent problème. La co-réflexion menée avec les opérateurs est guidée par la recherche d'alternatives pour une transformation du travail qui puisse soutenir le développement de l'activité des opérateurs et de leur santé de surcroit. Nous pensons que cette recherche d'alternatives doit se faire tant du côté des opérateurs que du côté des concepteurs impliqués dans le travail des opérateurs. Nous nous efforçons alors de proposer un cadre qui puisse interroger indirectement différents niveaux hiérarchiques et différents métiers au sein de l'entreprise, en passant toujours par le travail quotidien des opérateurs, afin de contribuer à la santé de chacun. C'est pourquoi l'analyse des conflits d'activité fait également l'objet de discussion entre concepteurs et managers concernant les choix de conception et d'organisation du travail à envisager à l'issue de l'intervention : la dimension prescriptive du travail des opérateurs, comme l'activité des commanditaires (concepteurs et/ou managers) 
font partie intégrante de notre analyse. Ainsi concepteurs et managers peuvent à leur tour découvrir d'autres voies possibles dans le travail et son organisation quotidienne, puis faire des choix de transformations du travail à partir des analyses réalisées sur le travail. Nous avons d'ailleurs remarqué que progressivement les dialogues au sein de l'instance de pilotage de l'intervention se réorientaient vers d'autres préoccupations que celles de la biomécanique des gestes sans pour autant en exclure leur importance : «comment peut-on travailler autrement [en conception] ? », " comment intégrer le travail des opérateurs dans le processus de négociation avec le client? » en reprenant les mots du commanditaire. Au cours des réunions du comité de pilotage, les discussions se sont orientées sur la manière dont il serait possible de mettre en place des instances d'analyse du travail à différents niveaux dans l'entreprise pour faire progresser leur organisation du travail au quotidien. Ce processus de transformation de la commande a pu être possible grâce aux ressources déployées du côté des opérateurs dans un premier temps - lors de la co-analyse du travail puis du côté des concepteurs-décideurs - lors des réunions de pilotage. Le cadre d'intervention a donc été co-construit à travers deux instances constituées spécialement pour le temps de l'intervention : un collectif d'opérateurs et un comité de pilotage.

Dans cette intervention, nous avons aussi pu faire l'expérience que les dialogues centrés sur l'analyse des gestes professionnels des opérateurs se sont avérés porteurs de nouvelles perspectives tant pour le travail quotidien de production que pour le travail de conception des lignes de production. Cette première description de l'intervention nous amène maintenant à analyser précisément les réels développements qui ont pu se produire, à en proposer une formalisation et enfin à poser les limites des effets produits.

\section{3.- De la fonction psychologique du collectif d'opérateurs à sa fonction sociale dans l'entreprise}

Notre perspective a pour objet d'action et de recherche le développement de l'activité (Clot, 2008b). Elle vise à seconder l'action de transformation de l'activité et à remettre le métier en mouvement au sein des collectifs de travail jusqu'à la transformation de la tâche. L'activité n'est donc pas seulement un objet d'étude, mais un instrument clinique décisif. Intervenants et professionnels travaillent ensemble pour prendre soin du métier et de l'organisation du travail (Clot, 1999 ; Clot, Faïta, Fernandez, \& Scheller, 2000 ; Scheller, 2001). Une analyse située de l'activité de travail subjective et collective, menée avec des opérateurs volontaires, est un premier pas essentiel pour questionner les prescriptions et l'organisation du travail de l'entreprise. Face aux questions de santé des opérateurs, cette intervention avait pour préoccupation centrale la question suivante : comment faire diffuser, déplacer les problèmes soulevés par la co-analyse du travail à différents niveaux dans l'entreprise ? Le travail de coanalyse avec les opérateurs comme celui en comité de pilotage font l'objet de la même attention clinique.

\section{3-1.- Organiser la confrontation entre opérateurs}

Dans cette première phase de l'intervention, nous nous sommes efforcés de construire une analyse construite avec une dizaine d'opérateurs majoritairement intérimaires. Le travail collectif des opérateurs pour réaliser la production est une injonction qui se trouve simultanément empêchée par le recours massif à l'intérim et un important turn-over (ex : chaque semaine entre au minimum un nouvel intérimaire dans une équipe de 12 à 15 opérateurs). Nous avons ainsi découvert un milieu de travail où apparaissaient des problèmes de santé - restants néanmoins « tabous » par la peur de perdre son emploi - avec d'importants conflits interpersonnels entre opérateurs. Face à ces difficultés naissantes dans l'entreprise, il a été proposé d'accompagner la restauration d'un collectif de travail malgré un contexte plutôt défavorable. La difficulté majeure de cette intervention repose sur les possibilités d'une réflexion sur le travail avec majoritairement des intérimaires (précisément 6 
intérimaires sur les 10 opérateurs volontaires). Tout au long de ce travail d'intervention, une question a constamment été présente à notre esprit: est-ce possible d'intervenir malgré l'instabilité du groupe d'opérateurs et l'exigence temporelle de notre méthodologie ? Face à ces difficultés, nous avons tenté de tenir malgré tout le cadre de l'intervention auprès de ces intérimaires afin de mener une réflexion sur le métier et la santé.

La méthodologie de co-analyse du travail a été déployée en plusieurs phases (Figure 1) :

— une première phase d'observations (papier-crayon) afin d'amener les opérateurs à percevoir leurs manières de faire intra et interindividuelles, souvent non conscientisées (exemple de verbatim en début d'intervention : "mais c'est facile ce qu'on fait ", "on fait tous pareil », "notre travail c'est la fiche de poste »), et à susciter des demandes pour réaliser une analyse du travail ;

- une deuxième phase pour débuter le travail d'analyse hors situation de travail quotidienne avec une réunion de préparation et de constitution du groupe d'analyse pour déterminer ensemble les situations de travail qui feront l'objet d'analyse ;

- une troisième phase d'observations avec caméra pour constituer des outils d'analyse concernant 4 situations de travail ou gestes professionnels retenus par le groupe (le port du pare-chocs à l'encyclage, le contrôle du pare-chocs en début de ligne, le montage à deux opérateurs sur le même pare-chocs, le réapprovisionnement des pièces) ;

- une quatrième phase pour les séances en auto-confrontations simples puis en autoconfrontations croisées pour concrétiser la confrontation entre pairs à propos de gestes professionnels précis ;

— une dernière phase afin de synthétiser des éléments d'analyse du travail et de constituer un montage vidéo de quelques situations de travail ayant fait l'objet d'analyse, suivies des dialogues en auto-confrontations croisées; ce montage étant destiné d'abord à l'ensemble de leurs collègues puis aux membres du comité de pilotage.

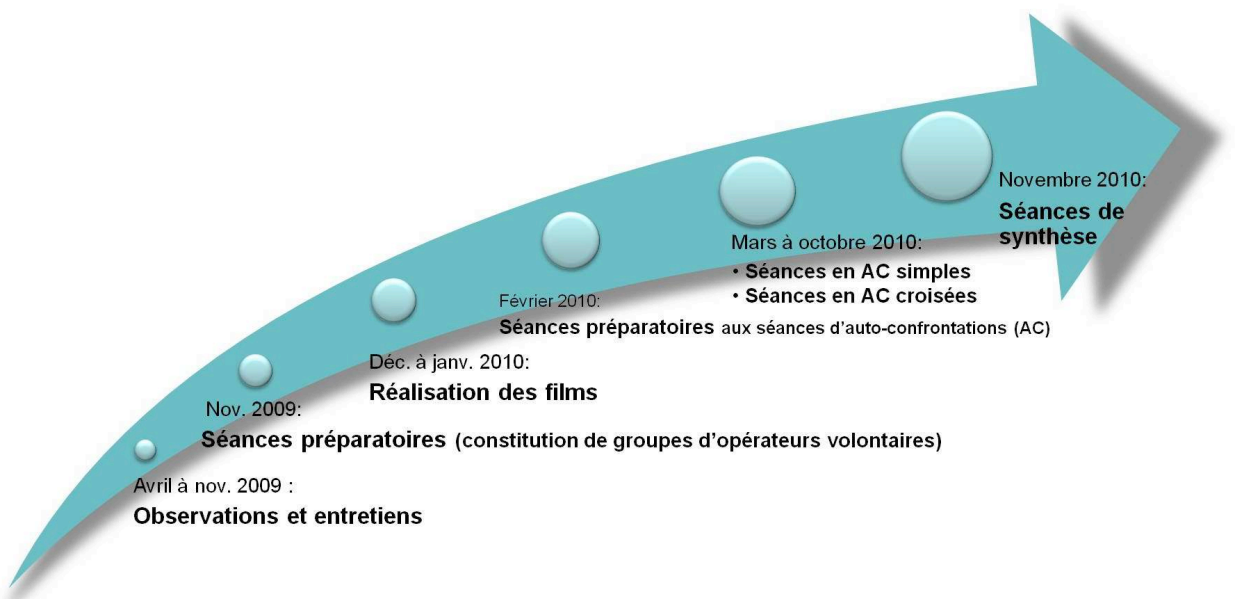

Figure 1 : Déroulement chronologique de la co-analyse avec les opérateurs

Figure 1: Operators co-analysis chronology

La co-analyse, telle qu'elle est conçue ici, propose à l'opérateur un dialogue différé sur les conflits réels de l'activité afin de découvrir la «palette » de ses différentes manières de faire et de celles de ses collègues. Ce dialogue différé reprend des réflexions plus ou moins partagées en cours d'activité ordinaire par les opérateurs. Il institue une comparaison systématique des manières de faire pour les transformer en objet de pensée préparant de nouvelles actions possibles. L'activité de l'un se réfléchit alors dans l'activité de l'autre. Le temps de l'analyse n'est qu'un temps spécifique pour « une transformation de l'expérience vécue et sédimentée en instrument pour vivre de nouvelles expériences » (Clot, 2008a, p. 148). Dans ce cadre, les opérateurs ont ainsi cherché à comparer leur activité réelle, à 
déceler les contrastes entre eux pour mieux modifier leurs propres façons de faire. C'est par ce processus que l'opérateur peut commencer à entrevoir les différentes possibilités qu'offre le collectif pour son activité individuelle. C'est pourquoi la centration sur l'activité concrète et les gestes quotidiens de travail font l'objet de préoccupation première pour la co-analyse du travail. En séance d'auto-confrontation croisée, les opérateurs peuvent re-découvrir dans leur activité des conflits irrésolus qu'il est nécessaire de «remettre en question » pour pouvoir continuer à travailler sans compromettre leur santé, voire même - et on suppose là un moyen de dépasser ces conflits - concevoir d'autres alternatives possibles en situation de travail. Ce questionnement autour d'alternatives possibles se réalise par la mise à distance avec ses propres gestes professionnels d'une part et par le regard du collègue d'autre part. Afin de montrer la fonction des dialogues sur un geste professionnel précis, nous proposons ci-dessous un court extrait d'une auto-confrontation croisée retranscrite entre une opératrice (C), un opérateur (S) et l'intervenante (I) sur la manière de contrôler le pare-chocs en début de ligne, au moment où ils regardent les images vidéo de $\mathrm{S}$ en train de contrôler un parechocs.

Pour précision, il s'agit d'une confrontation entre deux opérateurs reconnus comme experts du contrôle au sein de leur équipe. Avant le travail de co-analyse, les échanges entre ces deux opérateurs étaient devenus quasi impossibles face aux désaccords qu'ils ne parvenaient ni à mettre en commun ni à élaborer. Cependant au cours de leur engagement dans l'analyse de leur travail, ils ont finalement accepté de se confronter l'un à l'autre par la médiation de leur geste. Voici un extrait de cette auto-confrontation croisée :

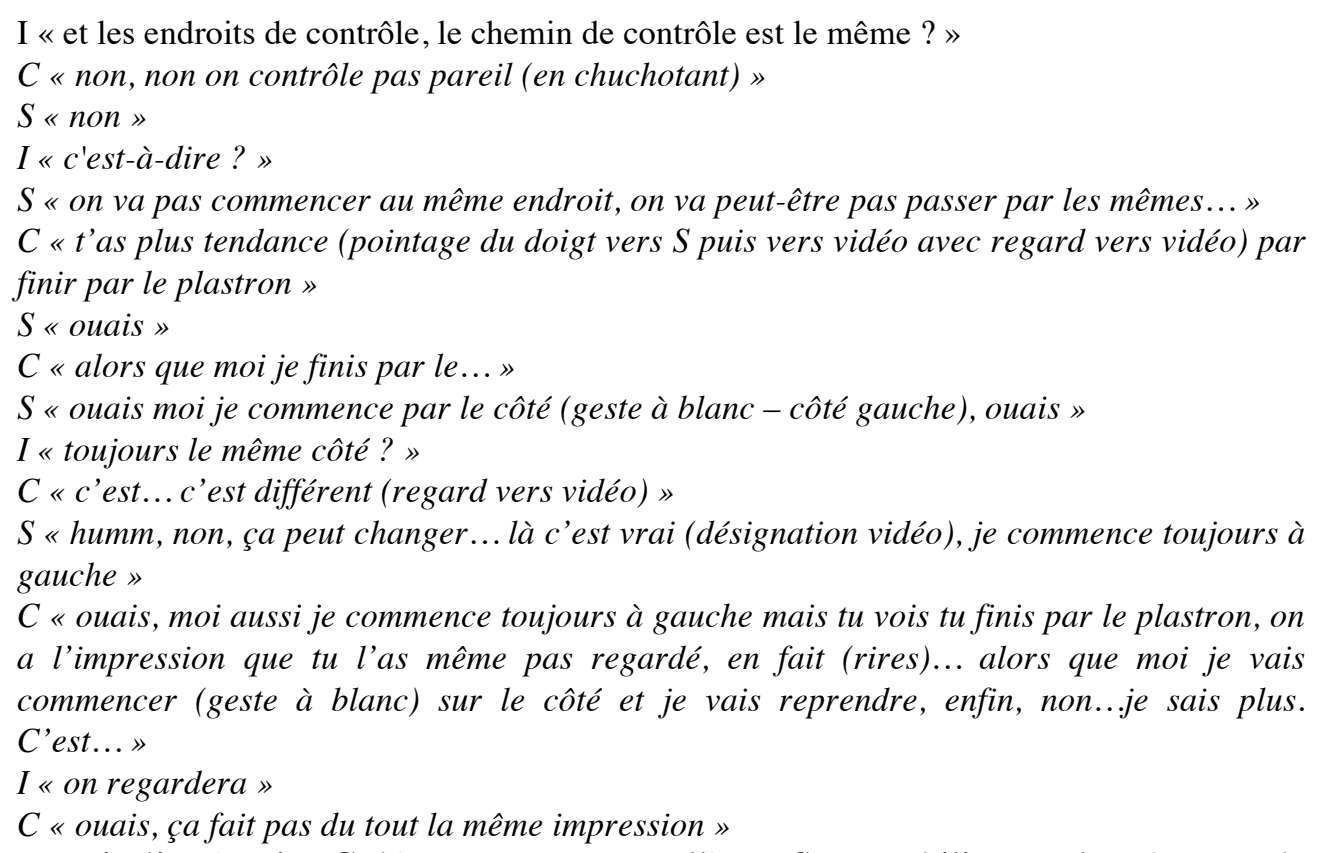

Dans cet extrait, l'opératrice $C$ découvre que son collègue $S$ ne mobilise pas le même mode opératoire alors même qu'ils travaillent quotidiennement dans la même équipe. L'observation précise des gestes de son collègue, à partir de la vidéo, la perturbe et l'empêche momentanément d'expliquer en quoi la façon de faire de son collègue est différente de la sienne. Du coup, elle finit par ne plus comprendre comment elle-même s'organise pour contrôler le pare-chocs. Ce moment de découverte des différences, subtiles mais essentielles, entre eux concernant un geste précis de leur travail, les renvoie à l'immensité du réel de l'activité et leur permet de se détacher de la procédure, le temps de l'analyse, pour y revenir plus tard. Ce point a été d'ailleurs débattu entre opérateurs, après les auto-confrontations croisées, pour prendre la décision de rendre visibles ou non leurs différences gestuelles lors du comité de pilotage. Il a été question de proposer un montage vidéo montrant une partie du film de montage des pare-chocs, suivie de dialogues controversés entre opérateurs manifestant ainsi, par le biais de leurs propres voix, leur prise d'initiatives sur les gestes professionnels, la complexité et la richesse de leur métier. Cet extrait de dialogue ci-dessus a finalement été retenu par les opérateurs et nous-mêmes dans 
le montage final à destination des membres du comité de pilotage. Le travail clinique avec les opérateurs est un moyen de développer leur propre activité au cours du temps, qui donne des possibilités d'agir sur soi et les autres. L'intervenante soumet aux opérateurs un questionnement qu'elle-même se pose : "comment allez-vous continuer ce travail réalisé ensemble? », "Pensez à la suite, à quoi ça va vous servir? », questions auxquelles il n'existe pas de réponses garanties d'avance mais qui pourraient leur ouvrir d'autres possibilités d'agir dans le travail quotidien à partir notamment de ressources collectives, jouant une fonction psychologique majeure pour la santé. Bien entendu ce travail de coanalyse n'est pas suffisant et ne se révèle pas comme la solution à tous les problèmes. De plus, nous ne sommes pas encore en capacité de déterminer si la poursuite et le maintien d'une co-analyse du travail au sein des équipes d'opérateurs pourraient se mettre en place dans la structure organisationnelle. Mais ces opérateurs ont fait l'expérience que «le collectif n'est jamais donné d'emblée, il est toujours à créer et à maintenir » (BournelBosson, 2010, p. 228). Même si les développements de l'activité et des relations professionnelles entre opérateurs, exposés ci-dessus, sont significatifs durant cette coanalyse - étant donné la précarité de leur statut dans l'emploi - ces développements restent fragiles et insuffisants s'ils ne sont pas relayés par les dirigeants et concepteurs de l'entreprise.

\section{3-2.- Organiser la confrontation entre opérateurs et concepteurs}

En parallèle de ce travail mené avec les opérateurs, un comité de pilotage a donc été mis en place durant l'intervention. Des réunions de travail ont été programmées à différents moments clés de l'intervention (Figure 2) : une première réunion de constitution du comité et de négociation des conditions d'intervention ; une deuxième réunion pour faire un point sur l'avancée de l'analyse et engager les discussions autour de la santé, la prévention et les procédures ; une troisième réunion de restitution du travail de co-analyse sous forme d'un montage vidéo construit avec les opérateurs et de négociation pour l'invitation des opérateurs pour une dernière réunion ; enfin une dernière réunion, non prévue au départ, afin de convier les opérateurs à la discussion sur la poursuite de ce travail. Cette dernière réunion a permis alors d'engager une réflexion collective sur l'élaboration d'une démarche d'intégration des opérateurs dans les projets de conception de l'entreprise.

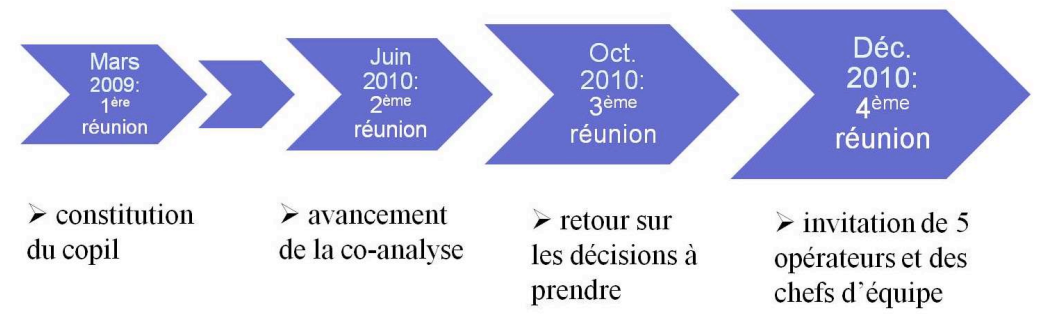

Figure 2: Chronologie des séances de travail en comité de pilotage

Figure 2: Steering committee work sessions chronology

Au fil des réunions du comité de pilotage, de plus en plus de personnes, tant au niveau opérationnel que fonctionnel (service qualité, études, commerce, etc.), ont été invitées ou ont demandé à y assister. Ce qui peut apparaître d'abord comme un risque de dispersion du travail engagé, s'est révélé au contraire un moyen de diffuser les questionnements abordés, avec précision et sans détour sur les alternatives possibles, notamment avec des membres de la direction locale et nationale. Dans cette instance de pilotage, nous retenons deux résultats importants : l'appel à une nouvelle commande sur le travail des chefs d'équipe, qui euxmêmes souhaitaient s'engager dans une réflexion sur leur métier; puis la décision d'organiser une autre réunion de pilotage en invitant des opérateurs ayant réalisé la coanalyse et leurs chefs d'équipes. Ainsi le déplacement qu'opèrent les membres du comité de pilotage grâce au travail accompli par les opérateurs permet de faire migrer la discussion de 
ces membres sur les gestes précis du travail quotidien des opérateurs, sujet qui leur était encore étranger. Nous retenons d'ailleurs un moment précis des dialogues lors de l'avantdernier comité de pilotage où la directrice du site prend la parole pour commenter le montage vidéo de la co-analyse présentée, "on voit qu'il y a eu du travail et c'est super intéressant de voir qu'au final les gens, ils ont différentes techniques, entre ce qu'on avait prévu sur les modes opératoires, les chemins de contrôle etc. Que finalement, les gens ne font pas pareil, mais le résultat au final, est qu'on a un résultat final en termes de qualité clients qui est vraiment bon.». À la fin de la réunion, elle proposera à ses collègues concepteurs une autre alternative de travail et de négociation avec le client: " je me dis aussi des fois, est-ce que ça vaut pas le coup de proposer (au client... qu') on souhaite faire avec les gens qui ont conçu les machines un retour d'expérience avec les opérateurs. Et nous, on leur propose ça, en leur disant ben voilà (...), on vous a fait un certain nombre de remarques mais maintenant, on en a d'autres avec l'expérience et, et c'est aussi l'occasion de repasser... ben voilà des messages, en disant ben voilà, on a besoin d'opérateurs, enfin avec les recherches que vous faites... ben voilà les gens ils ont besoin de pas avoir toujours le... la... les mêmes rythmes, de pouvoir faire les opérations... sur un autre puis... je pense que ça peut être constructif dans les autres projets quoi.»

Un autre moment de l'intervention en comité de pilotage peut faire l'objet d'analyse. Lors de la dernière réunion de l'instance de pilotage, cinq des dix opérateurs volontaires ont été invités à s'exprimer devant l'incertitude des membres du comité de pilotage pour améliorer leur façon de concevoir le travail des opérateurs. Lors d'un échange précis, juste après la diffusion du montage vidéo, le responsable projet (commanditaire principal), un opérateur $\mathrm{S}$, le responsable de production, une opératrice $\mathrm{C}$, un opérateur $\mathrm{J}$, la directrice et un chef d'équipe dialoguent sur la procédure concernant le chemin de contrôle visuel du pare-chocs (qui prévoit un parcours précis du haut - ce qu'ils nomment le plastron - aux côtés du parechocs). Voici l'extrait de cet échange. Nous citons l'ensemble de l'échange car sa longueur même est significative de l'engagement des différents interlocuteurs sur l'objet réel du travail :

Responsable Projets : "Je voudrais rebondir sur ce point-là, car moi il y a une question qu'on avait déjà évoquée la dernière fois mais qui m'interpelle, c'est moi avec la casquette projet [les fiches de poste] définissent un moyen, ben définissent une procédure, un fonctionnement, "on commence par le côté gauche, on fait ça et ainsi de suite" et on se rend compte par rapport à ce qu'on vient de voir et ce que vous évoquez, au-delà il y a des petits, des grands, des moyens, et ainsi de suite il y a quand même des façons différentes de fonctionner entre individus et moi je voulais profiter justement, parce que c'est pas forcément toujours le cas, de vous (les opérateurs) écouter... vous qu'on avait vu sur l'écran il y quelques minutes, vous pensez quoi de ses manuels, de ses instructions qu'on vous amène un peu toute faite, et quelque part vous interprétez, vous... qu'est-ce que vous en pensez de ça?»

Opérateur S : «[...] on va dire que c'est une base, comment eux ils veulent qu'on fonctionne mais après avec les critères des personnes qu'on a ici, ça peut marcher et avec d'autres ça peut pas marcher. Après c'est là l'interprétation qu'il faut avoir, il faudrait mettre en commun comme on a dit là pour voir la manière de faire... on va dire... parce qu'entre moi et $C$, on a pas la même façon et c'est... on va dire c'est la même chose en fait ».

Responsable production : «Est-ce qu'on peut dire que le résultat est le même au final ? »

Opérateur S : «Voilà, le résultat... on peut se baser sur les classeurs mais on a du mal à respecter à la lettre».

Opératrice C : "Ce serait plus dans le sens qu'il faudrait pas imposer le chemin, plus je pense... dans le sens... on va dire... identifier chaque partie du pare-chocs, le plastron, les arrêtes, la cross... je veux dire que les pare-chocs sont tous faits de la même manière, ils ont tous un plastron, des cross, des grilles, donc... après je pense que pour l'opérateur le fait d'avoir, lui dire ben voilà t'as le plastron, t'as le plastron à contrôler, t'as la cross, les 
arrêtes, bon... lui simplifier la tâche quoi entre guillemets, en disant il y a tel point, tel point à contrôler, t'as tous ses points à contrôler après tu t'arranges pour le faire dans le sens où tu le sens le mieux pour toi... que... ça laisse la personne libre du chemin qu'elle doit prendre et ce qu'elle a à regarder».

Directrice : "c'est plutôt donner une mission en disant voilà t'as ça, ça, ça à contrôler et c'est vous qui... la manière de...».

Opératrice $\mathrm{C}$ : «voilà comme ça, ça reste...»

Opérateur S : "peut-être les débuts... non, les formations on montre comment ça doit être fait à la lettre, après petit à petit la personne va nous voir comment nous on fait et elle inconsciemment elle va faire comme nous et au bout du compte ça marchera aussi bien que ce qui est demandé... et ça de savoir s'il y a possibilité si malgré les [fiches de poste], essayez d'avoir des petits changements comme ça, des petites solutions ».

Opérateur $\mathrm{J}:$ " ou de le faire en partenariat avec un opérateur »

Opérateur S : «voilà par exemple »

Directrice : « si chacun fait différemment il faut revoir [la fiche de poste] »

Chef d'équipe N : "Moi je pense le contraire... moi j’ai 2 formateurs de nuit, on doit avoir une base commune, parce que chez certains, j'ai déjà remarqué, c'est pas méchant mais si on les laisse travailler de la façon dont il le pense, il pense d'une certaine manière et ils sont pas toujours dans le vrai... parce que quand on est pris dans le travail et ben on a pas un regard objectif toujours sur ce qu'on fait et justement je trouve qu'on arrive vite à tout et n'importe quoi. Moi j'essaie surtout de fuir ça et de garder... parce que je pense que le chemin il est bien fait dans le sens où on commence par le plastron, c'est la zone principale et si on commence par les côtés et que le plastron n'est pas bon, ben le pare-chocs on peut l'ôter. Donc je pense qu'on gagne vachement de temps. Après les gens à un moment donné, on l'apprend pas de la même façon, c'est sûr que physiquement on a pas tous les mêmes bras (rires) ; c'est pas tout le monde qui est comme $S$, on dirait qu'il prend une feuille, voilà. Mais c'est vrai que sur les manières de prendre et de tout ça. Je comprends on a des tailles différentes, on essaie de faire des postes ergonomiques et tout mais moi je pense que sur les chemins de contrôle, les trucs comme ça, ça doit être hyper carré...»

Il y a ici, dans l'instance de pilotage, une poursuite de la discussion entre opérateurs puis avec un chef d'équipe sur la question des procédures et de la formation des nouveaux arrivés. La manière dont les opérateurs et les chefs continuent à développer le débat sur leur travail devient un moyen d'adresser leurs conflits d'activité, non plus seulement aux gens du métier, mais également aux concepteurs et dirigeants. Cet échange connait un destin favorable car dans la suite de la réunion, des discussions entre membres de l'encadrement opérationnel et fonctionnel ont lieu. Chacun tente, en partant de sa fonction dans l'entreprise (conception, achat, commerce, RH, etc.), de poursuivre plusieurs pistes. Pour nous, en tant qu'intervenant, l'objectif n'est pas de trancher la question mais plutôt d'accompagner le développement de ce dialogue, de ses protagonistes comme de ses objets afin d'identifier de nouvelles façons de concevoir le travail et de le réaliser, sans contourner le réel et les problèmes qu'il pose. Ainsi au sein du comité de pilotage, les opérateurs présents, préparés en amont par ce travail collectif d'analyse, font davantage autorité dans leur travail et du coup augmentent leur légitimité dans le travail des concepteurs et des managers. Faire autorité dans le travail n'est pas l'équivalent d'avoir le pouvoir sur le travail, ni même de posséder le pouvoir de décider, bien sûr, mais permet de ré-instruire le processus de décision. Cette préoccupation entre en écho avec les travaux en ergonomie sur la conduite de projet (Daniellou \& Martin, 2007) et notamment ceux de Béguin (2010) qui portent sur la rencontre de «mondes professionnels » où des professionnels de différents métiers doivent créer une forme commune de dialogue pour concevoir ensemble un projet.

Les déplacements, qui se produisent tout au long de cette intervention entre les opérateurs et 
les concepteurs, transforment alors le dispositif même de notre intervention et conduisent à installer cette dynamique d'analyse du travail, à différents niveaux dans l'entreprise pour y trouver quotidiennement des solutions à expérimenter en matière de qualité du travail comme en matière de santé. Cependant, malgré une volonté clairement énoncée par le comité de pilotage de mettre en place des instances de dialogues sur les problèmes concrets du quotidien, rien n'est joué d'avance. En effet, lors des controverses sur le contrôle du parechocs, identifiées précédemment, il se réalise seulement une levée partielle du déni installé quant au conflit de critères sur la qualité du travail et de la relation au client. Malgré et peutêtre en raison même des déplacements produits dans les dialogues entre opérateurs et concepteurs, il pèse des incertitudes sur la pérennité des instances d'élaboration de nouveaux compromis dynamiques sur le travail.

Durant l'intervention, nous distinguons finalement deux temps qui signalent, pour reprendre la conceptualisation de Vygotski, une «migration fonctionnelle » (Vygotski, 2003) ouvrant sur le développement de la fonction du collectif :

— le temps de la co-analyse où les opérateurs ont fait l'expérience de la fonction psychologique du collectif comme ressource pour l'activité individuelle;

— le temps de l'instance de pilotage où le collectif des opérateurs a pris une autre place dans l'entreprise et a assuré une fonction sociale nouvelle dans l'organisation, place qui mérite d'être regardée comme une ressource pour cette dernière.

Le collectif de travail assume donc en cours d'action une double fonction, psychologique et sociale. Et ce «nomadisme fonctionnel» (Clot, 2008a ; Kostulski \& Clot, 2007 ; Vygotski, 2003) est l'un des critères que nous retenons comme le signe de nouveaux développements favorables à la santé regardée comme production possible d'un nouveau pouvoir d'agir (Clot, 2008a). Cette extension du rayonnement de l'activité se trouve attestée dans les dialogues sur les conflits d'activité des opérateurs lorsqu'ils deviennent l'objet même de discussion entre concepteurs et managers. Les concepteurs ont ainsi fait l'expérience d'être affectés par le travail des opérateurs et s'autorisent alors à devenir eux-mêmes demandeurs d'une analyse de leur propre métier de concepteur. Cependant, la question de la pérennité des effets de l'intervention est loin d'être réglée et nous n'en sommes qu'à l'exploration et à l'inventaire de ses conditions dans notre recherche.

\section{4.- Trois conditions cliniques de l'intervention : objet de recherche}

À partir de ces résultats d'intervention, nous proposons une lecture analytique des conditions ayant favorisé les développements relevés précédemment. Dans notre recherche, la question de la transformation est fondamentale. Un des points centraux du travail de recherche sur l'intervention repose sur les questions suivantes: que cherche-t-on à transformer? Que transforme-t-on réellement? Ces questionnements viennent enrichir la manière dont les inattendus et les obstacles que nous rencontrons dans l'intervention peuvent devenir à leur tour d'autres moyens pour une intervention clinique de l'activité (Kostulski et al., 2011). Ainsi, à partir d'une intervention menée durant trois ans chez ce logisticien automobile, nous cherchons à contribuer au débat sur la place et la posture de l'intervenant clinicien de l'activité. On ne se focalise pas uniquement sur l'analyse du travail que nous produisons, mais sur la manière dont cette analyse, à laquelle les professionnels participent, sert de moyen pour ces mêmes professionnels d'alimenter les instances du métier (Clot, 2008a), dans l'objectif de revitaliser le travail et son organisation. Après ces actions auprès de cette entreprise, nous pouvons désormais tenter d'analyser ce qui a pu se produire pour que cette dynamique se réalise tant chez les opérateurs que chez les membres de l'instance de pilotage. En revenant sur le cadre de l'intervention qu'il fallait tenir et inventer dans le cours de l'action, les conditions ayant été favorables au développement de l'activité des 
professionnels seront davantage formalisées.

En partant des acquis de l'approche clinique de l'activité et la formalisation de repères méthodologiques pour mener à bien une co-analyse du travail (Duboscq, 2009), nous envisageons de proposer une formalisation des actions de l'intervenant et de celles des professionnels afin de mieux saisir les enjeux pour l'intervention et l'intervenant. En partant du principe qu'il importe de «nous méfier des évidences pour repérer ce qui semble de l'ordre du détail et relève en fait du révélateur » (Lacomblez, 2007, p. 2), nous tentons de formaliser quelques repères cliniques. Il s'agit alors de montrer en quoi notre «orientation générale $[\ldots]$ va au-delà des méthodes en ce qu'elle suppose une manière de se situer face à la complexité du réel, et une manière d'être et d'agir avec les autres en respectant les mondes professionnels» (Béguin, 2007, p. 378). Ainsi le repérage des conditions cliniques, qui accompagnent la technique méthodologique, nous apporte des pistes de recherche supplémentaires sur le développement de l'activité, des collectifs de travail et de leur fonction dans l'organisation du travail.

Cette tentative de formalisation ne représente en aucun cas une synthèse des conditions que requiert un travail clinique de l'activité en psychologie du travail mais présente quelques repères qui semblent jouer un rôle dans l'action. Trois conditions du travail clinique font l'objet d'intérêt au regard de cette intervention en particulier, mais également à travers l'expérience d'autres interventions : l'authenticité du dialogue sur le travail, la recherche de la controverse et la place laissée aux étonnements et aux affects. Ces conditions sont liées à l'action de l'intervenant et représentent un objectif sous-jacent: celui d'une « reprise en main » du dispositif par les acteurs de l'entreprise, opérateurs comme concepteurs.

Pour chacune de ces trois conditions, nous proposons une définition et une analyse pour tenter de mieux cerner ce qui a plutôt favorisé l'installation de ce cadre d'analyse si difficile à construire. La généralisation dans l'entreprise d'une co-analyse du travail pour progresser en matière d'organisation du travail par le développement d'un travail d'organisation délibéré est au cœur de nos préoccupations.

\section{4-1.- L'authenticité du dialogue sur le travail}

Pour rechercher un authentique «travail sur leur travail», une «vérité» du rapport dialogique qui ne triche pas avec le réel de l'activité (Clot, 2008a, p. 209 et suivantes), il est nécessaire de clarifier la méthodologie, ses enjeux et ses objectifs. Il est alors question d'échanger à propos de notre propre métier d'intervenant et de notre conception dialogique du travail, de l'activité et de la santé. Les opérateurs peuvent ainsi se projeter et imaginer ce que pourrait signifier pour eux « analyser le travail», en l'occurrence leur propre travail. Le cadre de confiance s'est construit entre autres par la création d'un espace de dialogues au quotidien, avec la présence régulière de l'intervenant dans l'atelier, où il est possible de parler «pour de vrai» de son travail, de ses «points aveugles», de ses «angles morts», c'est-à-dire aussi de ce qu'on cherche à faire sans y parvenir. Au début de l'intervention - ce qui est classique -, leur travail n'était «pas bien compliqué » d'après les opérateurs et ils faisaient «tous pareils». Il nous fallait alors dépasser ce discours convenu qui les empêchait de penser autrement leur travail, source de manifestations pathologiques (Clot \& Fernandez, 2005). Les interactions répétées de l'intervenante avec les opérateurs - en face à face, à deux ou en groupe -, avec une présence assidue du début à la fin de poste pendant plusieurs mois, a permis de tenir un registre original où les opérateurs ont eu la possibilité de sortir du silence leurs difficultés, leurs doutes, leurs interrogations sur leur travail. Il a été question de pouvoir discuter du travail comme ils n'avaient encore jamais « osé » en parler en révélant des dimensions insoupçonnées d'eux-mêmes. En effet notre manière de présenter notre démarche et notre discours «décalé », par rapport au discours hygiéniste sur la santé habituellement entendu et «intégré » par les opérateurs dans le milieu automobile, ont laissé entrevoir que d'autres façons de considérer son travail et sa santé semblaient possibles. $L a$ 
vérité de l'engagement de l'intervenante sur sa démarche et ses hypothèses concernant leur travail, la santé et le collectif peut permettre alors aux opérateurs, en retour, de s'engager authentiquement dans des dialogues sur leur travail où le difficile à dire l'emporte sur le déjà dit $^{1}$. Ainsi la présence assidue de l'intervenante dans la situation de travail n'est pas envisagée comme une manière de se «faire oublier» durant les observations, mais au contraire d'utiliser sa présence comme un outil méthodologique pour inciter les opérateurs à s'observer eux-mêmes (Simonet, Caroly, \& Clot, 2011). Notre objectif était de favoriser les échanges sur le travail et les gestes professionnels précis, d'abord par notre intermédiaire puis entre eux, afin de garantir des possibilités de développement de l'activité de chacun. C'est par ce processus que le travail de la demande s'est joué. Ce travail authentique réalisé par les opérateurs devait alors entrer en résonance avec l'activité des concepteurs. Lors du comité de pilotage, nous pensons que la diffusion du montage vidéo - en tant que présence différée des échanges authentiques des opérateurs sur les dilemmes de leur activité professionnelle - fait apparaître les opérateurs comme comptables et responsables dans le vif de l'échange. Ce déplacement provoqué a ensuite placé les concepteurs et dirigeants dans une zone de développement potentiel de leur activité propre et provoqué le dialogue entre eux.

\section{4-2.- $S$ 'engager dans la controverse}

L'engagement dans la co-analyse suppose également de pouvoir développer les désaccords avec les collègues opérateurs afin de pouvoir discuter sur les manières possibles ou non de régler les conflits d'activité, que chaque opérateur traverse dans son métier. Ainsi il est nécessaire pour les opérateurs de provoquer et de supporter la controverse avec ses collègues. La controverse est, d'après Litim (2006), « une activité où l'on cherche à convaincre l'autre », ce qui est à distinguer de deux autres formes de dialogue : la dispute et la discussion. En effet l'objectif des séances de travail, spécifiquement instrumentée en autoconfrontation croisée, est de chercher à convaincre son collègue que sa manière de faire «vaut le coup» et l'oblige à argumenter son point de vue. C'est pourquoi l'autoconfrontation croisée est indispensable au processus de co-analyse car en auto-confrontation simple, l'opérateur est seul avec l'intervenant. Ce dernier, n'étant pas «du métier», n'éprouve pas les conflits de l'activité comme un opérateur peut les vivre et peut plus difficilement contre-balancer les arguments de l'opérateur à propos de ses manières de réaliser son travail à partir des vidéos.

Malgré tout l'intérêt de la controverse pour le développement de l'activité, elle doit se faire dans un cadre réglé et «serein». Installer la controverse entre professionnels est sensible pour l'intervenant et souvent difficile à supporter pour les professionnels pour plusieurs raisons : parce que les conflits d'activité ne sont pas d'abord toujours conscients, que les relations interpersonnelles exercent une influence constante et que mettre «à nu» ses difficultés dans le travail perturbe sa propre activité (Kostulski, 2010). Ainsi notre intervention installe un cadre qui rend d'abord plus vulnérable puisqu'elle nécessite de se risquer à mettre à jour les désaccords avec autrui mais aussi avec soi-même à propos de l'activité. C'est pourquoi l'intervenant-psychologue doit redoubler de vigilance clinique pour installer ce cadre propice à supporter les déplaisirs de la confrontation, d'où peut sortir le plaisir de la découverte par le dialogue.

D'après nos recherches, la controverse semble être une condition favorable au développement de l'activité. Afin d'argumenter ce propos, nous prendrons l'exemple d'un dilemme non résolu pour les opérateurs qui a fait l'objet d'analyse : le temps de contrôle du

\footnotetext{
A ce propos, les notes prises par l'intervenante étaient utilisées dans les interactions avec les opérateurs. Ces derniers pouvaient lire les notes pendant les observations afin de pouvoir s'en saisir dans les discussions tant avec l'intervenante qu'avec leurs collègues.
} 
pare-chocs. Le dilemme repose essentiellement sur le fait qu'il faut aller suffisamment vite pour être en synchronisation avec la production des autres et respecter la procédure de contrôle exigeant 35 secondes de contrôle. L'opératrice $\mathrm{C}$, experte au contrôle pour sa rapidité et son efficacité à toute épreuve, est dans un premier temps déstabilisée de se voir «contrôler aussi vite» à la vidéo en auto-confrontation simple. Puis durant l'autoconfrontation croisée avec sa collègue $\mathrm{A}$ - qui remarque aussi sa rapidité - l'opératrice $\mathrm{C}$ est affectée par les remarques de A lui faisant part d'abord de son scepticisme sur sa manière de contrôler: "moi je trouve que t'axes pas assez sur le plastron (regard vers $C$ ). Après c'est peut-être que, t'as peut-être ton coup d'œil qui fait que, mais c'est vrai que tu prends pas quand même le...». L'opératrice A finit pourtant par énoncer un problème majeur dans leur activité quotidienne avec la présence quotidienne de nouveaux intérimaires : A «clac, clac... c'est toujours tac, tac, les pattes, oui c'est... que moi je vais faire le geste
quand même moins rapide. C'est peut-être aussi que (regard vers I)... je me sens peut-être
moins sécurisée que toi (regard vers $C$ )»

$C$ «'a peut-être ça»

A «mais (pointage vers vidéo) il est vrai qu'un nouveau (regard vers Co) qui te voit contrôler comme ça (geste de balancement de la main), c'est foutu »

C«oui, oui »

$A \ll$ c'est foutu»

$C \ll$ c'est clair»

$A$ «le mec, il contrôle pas le PC, il suit son mouvement et il contrôle rien. Parce que là il y a eu quand même des heures de...»

$C$ «bon après je les forme pas comme ça non plus » (rires)

A «j'espère... oui mais ils te voient, c'est toujours le même (haussement des épaules), c'est pareil, tu peux former d'une façon en disant regardes la procédure, c'est comme ça, comme ça mais ils te voient avant "attends la procédure, c'est quoi la procédure... elle, elle le fait, pourquoi pas moi" c'est ça le problème. J'ai vu des jeunes contrôler très vite, plus dans l'équipe à SA, quand j'étais passée, qui contrôlaient très vite, j'étais surprise. J'ai dit euh “ça fait longtemps que t'es là" "ça fait 15 jours" "d'accord”. Je vais pas dire qu'il contrôlait aussi vite que toi mais presque. Donc voilà ça me... ben $R$ entre autres, quand tu venais m'aider que SA n'étais pas là, (inaudible - main devant la bouche et baisse de ton)... bon c'est vrai que 35 secondes (=la prescription), c'est trop long mais ils vont dire tac, tac, tu regardes là...»

Pour accepter que l'opératrice A tienne ce discours sur sa façon de contrôler, il faut nécessairement un cadre de confiance mais aussi qu'elle accepte de pouvoir penser son activité autrement. Le fait que l'opératrice A mette finalement en discussion le problème de l'expertise de $\mathrm{C}$ face à la présence d'intérimaires, déstabilise d'abord sa manière de penser son travail et certainement son activité concrète de travail par la suite. Mais, après cette séance, ces deux opératrices reprendront sérieusement l'analyse de ce dilemme entre «vitesse et transmission du savoir-faire », avec leur hiérarchie de proximité. C'est pourquoi le risque qu'elles prennent en réinterrogeant leur propre activité concrète doit être repris à son compte par le collectif d'opérateurs d'abord puis dans l'organisation du travail ensuite pour tenter de résoudre ce conflit. Cette controverse notamment sur l'arrivée régulière de nouveaux intérimaires a de surcroit été une des préoccupations de la directrice et de la responsable des ressources humaines à la fin de l'intervention. C'est au moyen de la controverse, entre autres, que le cadre de la co-analyse devient ressource psychologique et sociale pour le collectif d'opérateurs.

Cette préoccupation d'une recherche constante de la controverse durant l'intervention n'a 
pas que des aspects techniques, comme le montrent les travaux de Roger (2007) avec les enseignants. En effet lors de l'analyse du travail, les professionnels peuvent être envahis d'étonnements, d'inquiétudes, de doutes, parfois même de culpabilité face à la confrontation avec soi-même ou avec son collègue. L'intervenant doit alors redoubler d'attention sur le cadre dialogique qu'il met en place. Le dispositif provoque un processus de déliaisonreliaison qui s'opère entre les 4 dimensions du métier: transpersonnelle, personnelle, interpersonnelle et impersonnelle (Clot, 2008a). Bien que déstabilisant puisqu'il vient en rupture d'équilibres parfois chèrement acquis, le contexte dialogique de l'intervention consiste à faire "migrer » une émotion, une pensée, une intuition personnelle émise par tel professionnel sur le registre transpersonnel du collectif de travail, pour mettre le genre professionnel au travail (Clot, 2008a). Ce processus n'exonère donc pas chaque professionnel de sa responsabilité propre et peut lui donner au contraire une disponibilité psychique nouvelle pour ressaisir son engagement. Prendre le risque de la confrontation permet de revitaliser le métier en s'y attaquant; en devenant l'interlocuteur du surdestinataire qu'il représente alors pour l'activité de chacun. Le collectif devient alors plus apte à s'expliquer avec le registre impersonnel, au demeurant indispensable, de l'organisation du travail. Le développement de l'activité se fait en sortant des «enveloppes » où elle a aussi trouvé momentanément à se stabiliser.

\section{4-3.- Étonnements et affects : l'activité transférentielle}

Dans ce cadre favorisant l'engagement de chacun dans un dialogue authentique sur le travail et centré sur les désaccords, un autre processus parait être en jeu : la place des étonnements et des affects dans l'analyse comme ressource pour développer sa propre activité. Les étonnements de l'intervenante lors des observations de la situation de travail ne sont pas ici pris au premier degré comme objet de recherche, mais comme moyen de provoquer la réflexion sur le travail chez les opérateurs. Non sans rapport avec la pratique clinique de l'ergonomie et même en s'y comparant, le clinicien de l'activité fait de sa présence un moyen de « dénaturaliser » la situation en inversant le statut de l'observateur. Autrement dit, en intriguant l'observé sur ce que l'observateur cherche à voir de son activité, il cherche à «développer l'observation de l'observé sur sa propre activité »(Clot \& Fernandez, 2005). Dans cette perspective, le questionnement fait place à l'expression de certains étonnements car «il s'agit moins de questionner pour obtenir une réponse définitive que d'ouvrir le champ des questions possibles pour l'observé lui-même.» (Simonet et al., 2011, p. 113). Nous avons cherché à «mettre à jour» nos étonnements comme moyen de co-analyser le travail des opérateurs et pour les utiliser dans le milieu de travail lors des interactions avec les opérateurs. Les étonnements de l'intervenant ne prennent pas leur source seulement dans les connaissances et des hypothèses scientifiques. Il peut être également affecté par ce qu'il observe ou ce qu'il ressent en situation de travail. Nous proposons alors d'étudier la manière dont les affects de l'intervenante ont parfois été non pas un obstacle mais une ressource pour l'action. Les affects peuvent ainsi devenir des instruments de travail dans l'intervention, tant ceux des professionnels que de l'intervenant. Précisément, les affects peuvent devenir moteurs s'ils ne sont ni négligés, ni maquillés pour s'abriter derrière la méthode. Dans le cas où nous acceptons d'être déstabilisés, dérangés par les affects ressentis durant le travail clinique dans l'entreprise, alors il nous faut trouver un moyen pour que ces affects élargissent tant le pouvoir d'agir des professionnels de l'entreprise que celui de l'intervenant. À la manière de Spinoza, on peut soutenir que l'effort pour augmenter le pouvoir d'agir n'est pas séparable d'un effort pour porter au maximum le pouvoir d'être affecté (Clot, 2008a, p. 26 et suivantes ; Deleuze, 1981).

Deux moments de l'intervention sont ici analysés afin de mieux comprendre le processus par lequel ce que nous appelons le pouvoir d'être affecté peut ouvrir sur le développement d'autres possibilités d'agir - possibilités souvent insoupçonnées aussi bien par les professionnels que par l'intervenant - : 
— La phase d'observations permet de vivre quotidiennement auprès des opérateurs afin d'installer durablement la co-analyse. Notre premier instrument de travail est de provoquer des étonnements sur notre façon de questionner leur travail. Précisément, lors d'une période où l'entreprise connaissait une production intense et une augmentation croissante du nombre d'intérimaires dans les équipes, un moment particulièrement déstabilisant pour l'intervenante a eu lieu. Au cours d'une journée de production, le chauffeur transportant les pare-chocs jusque chez le constructeur était absent et remplacé par un autre. Ce dernier était connu par les opérateurs comme étant «brutal» et ne prenant pas soin des pare-chocs lors du chargement dans le camion. En fin de journée de production, il a réalisé une mauvaise manipulation et a renversé un chariot complet de pare-chocs. À ce moment précis, l'intervenante a ressenti un « haut-le-cœur ». Ce malaise s'est amplifié étant donné les réactions du chef d'équipe et des opérateurs : ces derniers ont alors pris « un air blasé » et se sont mis dans la foulée à refaire la production de toute urgence. L'intervenante décide ensuite de partager son émotion avec les opérateurs quant à la situation et son étonnement face à leurs réactions. Ils se sont étonnés en retour de cet « éprouvé » et se sont mis à en débattre entre eux en questionnant alors la valeur même de l'objet matériel travaillé : le pare-chocs. À partir de ce moment-là, on a observé des changements dans les échanges avec l'intervenante et entre eux : ils se sont mis à parler plus aisément du pare-chocs. Des discussions sur le ton de l'humour ont eu lieu par la suite autour de cet objet: "t'aimes pas quand ça choque le pare-chocs!» ou «ça te choque que le pare-chocs choque» expressions adressées alternativement tant à l'intervenante qu'aux collègues; ou encore des discussions autour des modèles ou couleurs qu'ils préfèrent: "c'est mon bleu, tu fais attention... oui les bleus, c'est mes préférés » dit une opératrice à son collègue saisissant le pare-chocs de couleur bleu qu'elle venait de contrôler. Des moments comme celui-ci, où l'affect éprouvé devient un moyen de transport de l'échange entre intervenant et opérateurs ont été saisis par eux comme un moyen indirect de se rapprocher de l'objet travaillé plus librement que ne l'autorise le genre de discours convenu dans l'atelier. Ce qui est aussi l'occasion d'identifier pour eux les préoccupations réelles de l'intervenant autour du travail «bien fait ». C'est donc à travers la possibilité de mobiliser les affects éprouvés au cours de cet événement potentiellement banalisé, que les opérateurs parlent finalement, non sans humour, des façons de «prendre soin » du pare-chocs. Il faut prendre toute la mesure de cette donnée clinique. C'est en effet l'une des modalités sous laquelle peut s'opérer ce qu'on peut appeler un transfert sur la méthode proposée en clinique de l'activité : dialogue et confrontation sur la qualité du travail. Cette circulation des affects comme moyen de transport de l'analyse du travail jusqu'à l'appropriation de la méthode dialogique invite l'intervenant à soigner cette activité transférentielle. Son maniement déborde, comme le montre cet exemple, l'espace-temps de l'autoconfrontation. Il transite aussi par une conception clinique de l'observation directe en situation. Ces moments à saisir font éventuellement et momentanément perdre à chacun sa «contenance ${ }^{2}$, tant pour les opérateurs que pour l'intervenant, mais ils paraissent fournir un moyen puissant pour « faire parler le métier » dans des organisations qui ne s'y prêtent guère.

- Une autre situation permet également d'étayer le rôle des affects dans l'intervention. Durant l'organisation et la mise en place des séances d'analyse avec les opérateurs, garantir la présence des intérimaires du début à la fin du processus d'analyse est devenu impossible. Face aux départs de plusieurs intérimaires, investis pleinement dans le travail de réflexion, nous avons d'abord ressenti des affects de colère puis de résignation devant le fait de ne plus pouvoir installer un travail clinique sur le travail. Afin de ne pas

2 C'est peut-être la définition même de l'affect dans l'activité, au-delà de l'émotion qui n'est jamais que son instrument extérieur (Clot, 2008a, p. 8). L'affect est le déphasage vital qui surgit entre les attendus habituels du sujet, ses organisateurs préconçus (qu'ils soient corporels, cognitifs ou subjectifs) et les inattendus de l'activité en cours (Article à paraître). 
refermer le cercle de notre activité sur ces affects mais plutôt de tenter de l'ouvrir grâce à eux, nous avons pris la décision de faire part au commanditaire de notre inquiétude sur la qualité de notre travail d'intervention dans de telles conditions. Quelque temps plus tard, nous avons appris que des recrutements en CDI de plusieurs opérateurs intérimaires de la cellule «pare-chocs » étaient en cours. Dans un premier temps et par prudence, nous n'avons pas fait le lien avec notre intervention. Mais une rencontre fortuite avec la directrice du site nous a permis d'émettre l'hypothèse suivante: notre manière de déplacer le problème de l'intérim dans un autre contexte, l'avait sans doute «amplifié » en rendant visibles d'autres conséquences que celles que l'organisation a apprises à " gérer », affectant à leur tour l'activité des dirigeants et concepteurs et sans doute leurs échanges. À notre adresse, la directrice du site formule alors les choses ainsi : «vous avez $v u$, on a recruté, ça va être bien pour votre étude ». La question de l'intérim n'a plus été seulement le problème de l'intervenante, des équipes d'opérateurs et de leur hiérarchie de proximité, mais elle s'est trouvée chargée de significations nouvelles pour les dirigeants, au moins dans ces circonstances. La prudence exige de ne pas faire d'imputations causales simplistes dans ce genre d'interactions. Disons simplement que l'engagement dans notre intervention, au nom de la qualité de notre propre travail, a pu s'ajouter aux motifs de la décision prise.

Le but de l'intervention a été d'expérimenter la fonction du collectif dans l'activité quotidienne de travail. Mais comme nous avons pu le constater, la dynamique mise à jour est imputable à une technique de co-analyse se prêtant délibérément aux transports des affects auxquels l'intervenant doit non seulement être attentif mais veille à les «enrichir». Ce dernier utilise ainsi ses affects pour seconder le travail sur les différents registres du métier et leurs conflits, du point de vue des opérateurs et du point de vue des commanditaires, les premiers ne s'autorisant parfois plus à faire «parler le métier» dans ce qu'il a de plus personnel et transpersonnel, les autres s'étant parfois affranchis de leur fonction propre sur le registre impersonnel de l'activité considérée.

\section{5.- Discussion : vers quel modèle de la transformation en clinique de l'activité ?}

Partir d'une intervention permet de mieux saisir les enjeux méthodologiques et surtout cliniques. Il paraît important de rendre compte de nos manières d'intervenir afin d'engager un dialogue entre spécialistes de l'intervention en santé-travail, une nécessité tant pour les praticiens que pour les chercheurs comme le rappellent F. Hubault et F. Bourgeois (2004). En cherchant à analyser les conditions cliniques favorisant le développement du pouvoir d'agir sur les situations de travail par les opérateurs d'abord puis par les membres du comité de pilotage ensuite, nous souhaitions contribuer à la formalisation de nos actions et permettre ainsi de développer l'efficacité de nos interventions à venir. D'abord une question pour l'action, c'est aussi une question pour la recherche. Comme G. Devereux le note, « une science du comportement authentique existera quand ceux qui la pratiquent se rendront compte qu'une science réaliste de l'humanité ne peut être créée que par les hommes qui sont le plus conscients de leur propre humanité, précisément lorsqu'ils la mettent le plus totalement à l'œuvre dans leur travail scientifique. »(Devereux, 1980, pp. 20-21). Nous pouvons épauler cette position épistémologique par le propos de M. Bakhtine : «Dans les sciences humaines, l'exactitude consiste à surmonter l'altérité de ce qui est autre sans le transformer en quelque chose qui est à-soi » (Bakhtine, 1978, p. 392).

Le travail clinique exposé ici est un moyen de développer l'activité des professionnels. La centration sur l'activité concrète est essentielle et passe par l'analyse, précise et sans détour des gestes quotidiens de travail. C'est l'objet de préoccupation majeure pour l'analyse du travail que nous menons. Il y a aussi un décalage certain mais nécessaire, que nous devons mettre en tension, entre le développement des personnes, du collectif et de l'organisation du 
travail. Cette intervention montre comment des responsables de l'entreprise sont parvenus à envisager différemment les problèmes de santé en s'interrogeant sur leur conception des changements, sur "la façon de les mener en s'affranchissant de l'indispensable travail d'organisation que réalisent les salariés pour réussir à travailler correctement dans la durée » (Clot, 2010, p. 103). Reste à prendre la mesure des transformations que l'intervention peut produire, qui nécessite un engagement continu de chacun dans l'entreprise. Ce travail de suivi va pouvoir se poursuivre. Une intervention auprès des chefs d'équipe est actuellement en cours et certains concepteurs «méthodes et études » ont récemment été demandeurs d'une analyse de leur propre métier. Mais nous n'échappons pas à la question des limites de notre action alors même que nous sommes seulement des intervenants extérieurs.

La dynamique d'un collectif de professionnels est traversée par des échecs, des réussites, des joies, des peines qui peuvent être aussi vecteurs de santé quand ces professionnels tiennent le cap de la prise d'initiatives sur leur travail comme ont pu en faire la preuve ces opérateurs intérimaires, et ce malgré les conditions défavorables à sa construction. Nous retenons un moment précis des auto-confrontations croisées où un opérateur intérimaire propose à l'intervenante, face à son collègue embauché en CDI, de trouver d'autres moyens de faire l'analyse du travail. Il cherche à développer le cadre de co-analyse, de pallier les difficultés qu'il ressent avec seulement la vidéo comme support d'analyse. Il propose alors à l'intervenante de «le faire en direct », en situation concrète de travail, avec l'ensemble du collectif d'analyse, pour tester les différentes manières de porter le pare-chocs à l'encyclage. Les destins possibles de l'activité de chaque opérateur dépendent tant du collectif de travail que de l'opérateur lui-même, avant d'être déterminés par les managers et dirigeants. Expérimenter ce dispositif de co-analyse amène les opérateurs à prendre conscience de la fonction du collectif dans leur propre activité. Puis leur participation au dernier comité de pilotage est l'occasion pour eux de découvrir comment leur place - en tant que collectif d'opérateurs - peut se transformer au sein même de l'entreprise. Ainsi ils ont fait l'expérience, alors qu'ils n'étaient qu'intérimaires pour la plupart, de la fonction qu'a pu prendre le collectif pour eux-mêmes et pour l'entreprise (Quillerou-Grivot, 2011). C'est pourquoi nous avons cherché à instituer un cadre de pilotage de l'intervention où il a été question de débattre de la conception et sur les choix à faire à partir du travail réflexif des opérateurs, non pas comme contrainte mais comme ressource dans l'activité des concepteurs. Mais cette dimension de l'intervention reste à explorer tant du point de vue théorique que méthodologique. En effet, de nombreuses questions se posent sur l'activité des concepteurs en comité de pilotage, comme sur celle de l'activité des opérateurs quand ils sont amenés à être confrontés au «monde professionnel» des concepteurs (Béguin, 2010). L'action en clinique de l'activité dans l'instance de pilotage et son retentissement au sein de l'organisation du travail reste une question ouverte, tant du point de vue de son efficacité à long terme (par la mise en place de réelles transformations du travail et des effets sur la santé) que du point de vue de sa formalisation théorique. D'autres recherches-interventions sont en cours de développement.

Le choix d'exposer ce qui a permis de développer l'activité des opérateurs et des commanditaires (concepteurs et managers) le temps de l'intervention laisse encore en suspens certaines questions notamment sur la pérennité de ces développements produits après l'intervention et de leurs effets pour la santé à long terme. Après l'intervention, notre travail co-construit doit trouver d'autres garanties et être réinventé par les acteurs de l'entreprise. Nous retenons deux temps forts de la fin d'intervention qui tracent de potentielles transformations de la situation de travail des opérateurs : la proposition d'un opérateur intérimaire de continuer l'analyse « en direct » avec ses collègues et la décision du comité de pilotage d'inviter des opérateurs lors des rencontres avec le client pour un nouveau contrat. La proposition d'un des opérateurs est significative de l'expérience que les opérateurs ont acquise en faisant davantage autorité dans leur travail. Ils sont mieux placés pour défendre leur métier auprès de leur hiérarchie en raison du fait qu'ils s'y sont d'abord 
attaqués entre eux. La décision d'organiser le travail du comité de pilotage tient sans doute aux nécessités de faire place à une exigence marquée dans des travaux de plus en plus nombreux : l'institution d'une « instance » de ce genre dans l'entreprise permet d'instruire la controverse sur les critères du travail de qualité entre tous les partenaires sociaux, opérateurs, dirigeants et concepteurs (Clot, 2010 ; Ferreras, 2012 ; Petit, Dugué, \& Daniellou, 2011 ; Segrestin \& Hatchuel, 2012). Ce type d'approche, qui met en rapport les recherches en psychologie du travail et les recherches en sciences de gestion pour favoriser un travail d'organisation conjoint sans oblitérer les conflits (Bertrand \& Stimec, 2011 ; Detchessahar, 2011; de Terssac, 2002), expérimente une modification du système des relations professionnelles. Mais il nous éloigne aussi des illusions qui ont cimenté le courant des «relations humaines » en psychologie industrielle depuis l'expérience Hawthorne (Illouz, 2006 ; Mayo, 1945). Il mériterait d'être systématiquement expérimenté et évalué. Nous y voyons là une question à laquelle doit se mesurer la clinique de l'activité en psychologie du travail. C'est peut-être aussi un problème auquel tous les intervenants dans les organisations sont maintenant confrontés.

\section{BIBLIOGRAPHIE}

Bakhtine, M. (1978). Esthétique et théorie du roman. Paris: Gallimard.

Béguin, P. (2007). Dialogisme et conception des systèmes de travail. In Y. Clot \& K. Kostulski, Dialogue, activité, développement. Psychologie de l'interaction, $\mathrm{n}^{\circ} 23-24$ (pp. 169-198). Paris: L'Harmattan.

Béguin, P. (2010). Conduite de projet et fabrication collective du travail : une approche développementale. HDR en ergonomie. Université Victor Segalen Bordeaux 2.

Bertrand, T. \& Stimec, S. (2011). Santé au travail. Voyage au pays du Lean Management. Revue française de gestion, 5/ 214, 127-144.

Bournel Bosson, M. (2010). Analyse du travail et revitalisation du collectif. In Y. Clot \& D. Lhuilier (Eds.), Agir en clinique du travail (pp.225-236). Toulouse: Eres.

Clot, Y. (1999). La fonction psychologique du travail. Paris: PUF.

Clot, Y. (2005). Une intensification du travail peut-elle en cacher une autre ? In P. Askenasy, D. Cartron, F. De Coninck, \& M. Gollac (Eds.), Organisation et intensité du travail (pp. 313-317). Toulouse: Octarès.

Clot, Y. (2008a). Travail et pouvoir d'agir. Paris: PUF.

Clot, Y. (2008b). La recherche fondamentale de terrain : une troisième voie. Education Permanente, $\mathrm{n}^{\circ} 177,4$.

Clot, Y. (2010). Le travail à cœur. Pour en finir avec les risques psychosociaux. Paris: La Découverte, Cahiers libres.

Clot, Y., Faïta, D., Fernandez, G. \& Scheller, L. (2000). Les entretiens en auto-confrontation croisée : une méthode en clinique de l'activité. Pistes, 2(1).

Clot, Y., \& Fernandez, G. (2005). Analyse psychologique du mouvement : apport à la compréhension des TMS. TMS. Activités, 2(2), 69-78, http://www .activites.org/v2n2/fernandez.pdf

Daniellou, F., \& Martin, C. (2007). La formalisation de l'intervention en ergonomie, des contextes et des rencontres. Education Permanente, $\mathrm{n}^{\circ}$ 170, 2007-1, 63-75.

Deleuze, G. (1981). Spinoza. Philosophie pratique. Paris: Éditions de Minuit.

Detchessahar, M. (2011). Santé au travail. Quand le management n'est pas le problème mais la solution. Revue française de gestion, 5/ 214, 89-105.

Devereux, G. (1980). De l'angoisse à la méthode dans les sciences du comportement. Paris: Flammarion.

Duboscq, J. (2009). Développement de la sécurité et activités médiatisantes. Le cas du travail de gros-œuvre sur les chantiers de bâtiment. Thèse de doctorat en psychologie. Paris: CNAM.

Ferreras, I. (2012). Gouverner le capitalisme ? Paris: PUF.

Hubault, F., \& Bourgeois, F. (2004). Disputes sur l'ergonomie de la tâche et de l'activité, ou la finalité de l'ergonomie en question. Activités, 1(1), 34-53, 
http://www .activites.org/v1n1/vol1 num1.book.pdf

Illouz, E. (2006). Les sentiments du capitalisme. Paris: Seuil.

Kostulski, K. (2010). Quelles connaissances pour l'action en clinique du travail ? Une contribution à la question des rapports entre les actions de transformation et la production de connaissances en clinique du travail. In Y.Clot, \& D. Lhuilier (Eds.), Agir en clinique du travail (pp. 27-38). Toulouse: Eres.

Kostulski, K., \& Clot, Y. (2007). Interaction et migration fonctionnelle : un développement en autoconfrontation croisée. Psychologie de l'interaction, $\mathrm{n}^{\circ}$ 23/24, 73-108.

Kostulski, K., Clot, Y., Litim, M., \& Plateau, S. (2011). L'horizon incertain de la transformation en clinique de l'activité : une intervention dans le champ de l'éducation surveillée. Activités, $8(1)$, 129-145, http://www .activites.org/v8n1/v8n1.pdf

Lacomblez, M. (2007). Le « eux » / « elles » est-il de mise en ergologie? Article pour le séminaire «Tâches du Présent ». 10 et 11 mars 2007, Aix en Provence.

Litim, M. (2006). Les histoires racontées au travail. Métier et activité dans un service de gériatrie. Thèse de doctorat en psychologie. Paris: CNAM.

Litim, M. (2012). Les méthodes indirectes à l'épreuve de la pratique : questions d'intervention. In Y. Clot (Ed.), Vygotski maintenant (pp. 165-175). Paris: La Dispute.

Mayo, E. (1945). The Social Problems of an Industrial Civilization. Andover: Harvard University.

Petit, J., Dugué, B., \& Daniellou, F. (2011). L’intervention ergonomique sur les risques psychosociaux dans les organisations : enjeux théoriques et méthodologoiques. Le Travail Humain, 74(4), 391-409.

Quillerou-Grivot, E. (2011). Fonction psychologique et sociale du collectif pour la santé au travail : le cas d'opérateurs de montage automobile. Thèse de doctorat en psychologie. Paris: CNAM.

Roger, J.L. (2007). Refaire son métier. Essai de clinique de l'activité. Toulouse: Eres.

Sannino, A. (2012). Dialectique et intervention en théorie de 1'activité. In Y. Clot (Ed.), Vygotski maintenant (pp. 213-237). Paris: La Dispute.

Scheller, L. (2001). L'expérience du travail dans le cadre dialogique. Travailler, 6, avril 2001, 71-88.

Scheller, L. (2010). Transformations organisationnelles, conflits générationnels, clinique de l'activité : le cas d'un atelier industriel. Activités, 7(1), 62-74, http://www .activites.org/v7n1/v7n1.pdf

Segrestin, B., \& Hatchuel, A. (2012). Refonder l'entreprise. Paris: Seuil.

Simonet, P., Caroly, S., \& Clot, Y. (2011). Méthodes d'observation de l'activité de travail et prévention durable des TMS : action et discussion interdisciplinaire entre clinique de l'activité et ergonomie. Activités, 8(1), 104-128, http://www .activites.org/v8n1/v8n1.pdf

Terssac, de. G. (2002). Le travail, aventure collective. Toulouse: Octarès.

Vygotski, L. (2003). Conscience, inconscient, émotions. Paris: La Dispute.

Volkoff, S. (2008). L'intensification du travail "disperse" les problèmes de santé. In G. de Terssac, C. St-Martin, \& C. Thebault (Eds.), La précarité : une relation entre travail, organisation et santé (pp. 29-40). Toulouse: Octarès.

\section{RESUME}

L'objectif de cet article est de proposer, à partir d'une intervention chez un logisticien automobile, l'analyse de conditions favorisant le développement de l'activité, du métier et de la santé au travail. Le cadre de l'intervention en clinique de l'activité et sa méthodologie, déployés auprès d'opérateurs de montage de parechocs, nous amènent à tenter une formalisation de l'action du clinicien de l'activité qui cherche à transformer les obstacles organisationnels en nouvelles ressources pour le développement du métier d'opérateurs. Nous insistons sur la nécessité d'un travail clinique tant avec les opérateurs, par le biais d'une co-analyse du travail, qu'avec les concepteurs et décideurs de l'entreprise en comité de pilotage. Cette double visée de la recherche-intervention permet ainsi de maximiser les possibilités de développement de 
l'activité des opérateurs et de transformations de l'organisation du travail, malgré l'incertitude que laissent toujours nos actions face à la complexité de l'activité humaine et des organisations du travail. En partant du travail en intervention et des effets produits, nous proposons trois conditions cliniques qui ont semblé fondamentales pour permettre des développements de l'activité et un retentissement plus large au sein de l'organisation. Ces trois conditions renvoient à : l'authenticité du dialogue sur le travail, l'engagement dans la controverse, le transfert des étonnements et affects. Cette proposition de formalisation, au-delà de la méthode, permet d'alimenter le débat autour du modèle de la transformation lié à une approche clinique de l'activité.

MOTS-CLES

comité de pilotage, santé, co-analyse du travail, intervention, collectif du travail, automobile, développement de l'activité

\section{REFERENCEMENT}

Quillerou-Grivot, E., \& Clot, Y. (2013). Trois conditions pour une clinique de l'activité en psychologie du travail: le cas d'une intervention dans une entreprise de logistique automobile. Activités, 10(2), 229-248, http://www.activites.org/v10n2/v10n2.pdf

Article soumis le 3 janvier 2012, accepté pour publication le15 janvier 2013 